\title{
Magnetic resonance imaging of pelvic masses complicating pregnancy
}

\author{
PHILLIP R. LUBBERS, CDR, MC, USNR \\ CYNTHIA IZUNO, LT, MC, USNR \\ WALTER B. GOFF, CDR, MC, USN \\ ALAN JOHNSON, LCDR, MC, USNR \\ RANDLE SIKES, LCOL, MC, USA
}

The need for a safe, complementary examination to ultrasonography (US) in the obstetric patient with complications has been recognized. The safety of magnetic resonance imaging (MRI) has been demonstrated; to date, however, there have been relatively few reports of MRI in the obstetric patient. The authors examined three patients whose pregnancies were complicated by a pelvic mass with both US and MRI. The MRI examinations provided information that added to the US findings, and, in one case, MRI provided data that was not demonstrated on US. This experience, although limited, indicates that MRI can supplement the US examination in selected obstetric patients.

The discovery of a pelvic mass during pregnancy may have serious obstetric implications. ${ }^{1}$ The utility of ultrasonography (US) in the evaluation of pelvic masses in pregnancy is well known ${ }^{2,3}$; however, adequate sonographic evaluation may be hampered by overlying bowel gas, position of the mass in the pelvis, or body habitus.

Prior to the clinical use of magnetic resonance imaging (MRI), no safe, noninvasive, alternative technique was available to supplement the evaluation of these patients.

All three cases of pelvic mass complicating pregnancy reported here were evaluated with both US and MRI. MRI provided diagnostic or confirmatory information about the nature of the mass in each case.

\section{Report of case \\ MRI technique}

Three patients who were diagnosed as having a pelvic mass associated with pregnancy were evaluated at $\mathrm{Na}$ val Hospital Bethesda (NHB), Bethesda, Md, between
October 1985 and January 1986. Gestational age ranged from 15-22 weeks. Informed consent was obtained prior to the examination.

US was the initial imaging examination in each case. The sonographic evaluation was performed with a 3.5 $\mathrm{MHz}$ mechanical sector transducer with adjustable focal zone (Johnson \& Johnson).

The MRI evaluation was performed the same day as US for one patient, and four and eight days after US for the other two. Proton MRI was performed with a Technicare Teslacon system operating at $1.5 \mathrm{~T}$. T1-weighted $(\mathrm{TR}=700 \mathrm{msec}, \mathrm{T} 2=33 \mathrm{msec})$ and $\mathrm{T}-2$ weighted $(\mathrm{TR}$ $=2,000 \mathrm{msec}, \mathrm{TE}=100 \mathrm{msec}$ ) images were obtained using the spin-echo technique.

Imaging was performed in the transverse, coronal, and sagittal planes in two patients; one patient was studied in only the transverse and sagittal planes. Sequential, $0.5-$ to $0.8-\mathrm{cm}$ thick sections were obtained. Gap thickness with this machine is approximately $20 \%$ of the sec-

Fig 1. Transverse pelvic US: Cystic, cul-de-sac mass to the right of midline (arrow) contains internal echoes and septations ( $B L$ = bladder; case 1 ).

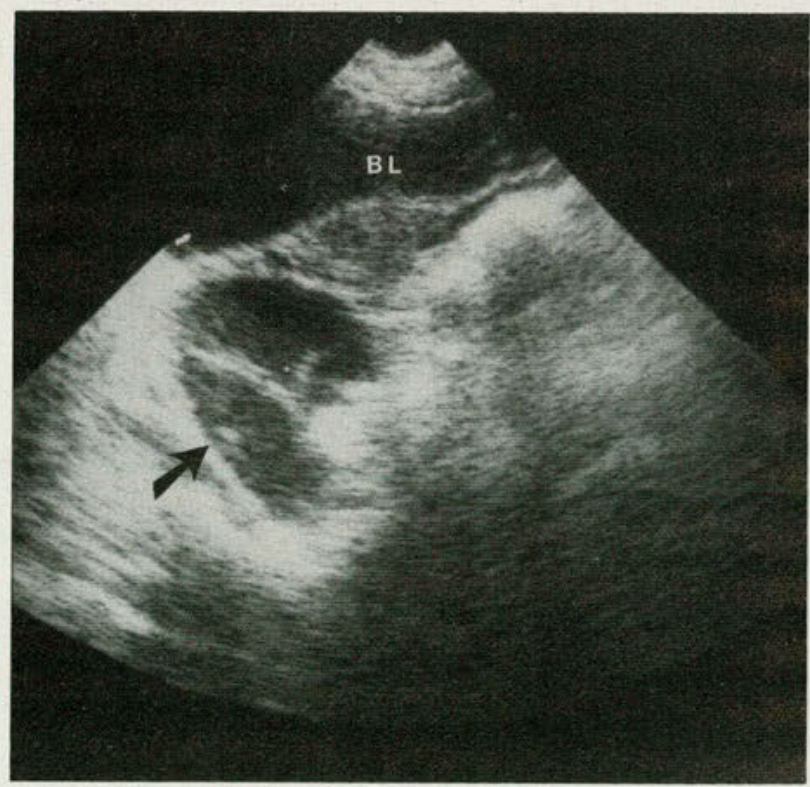




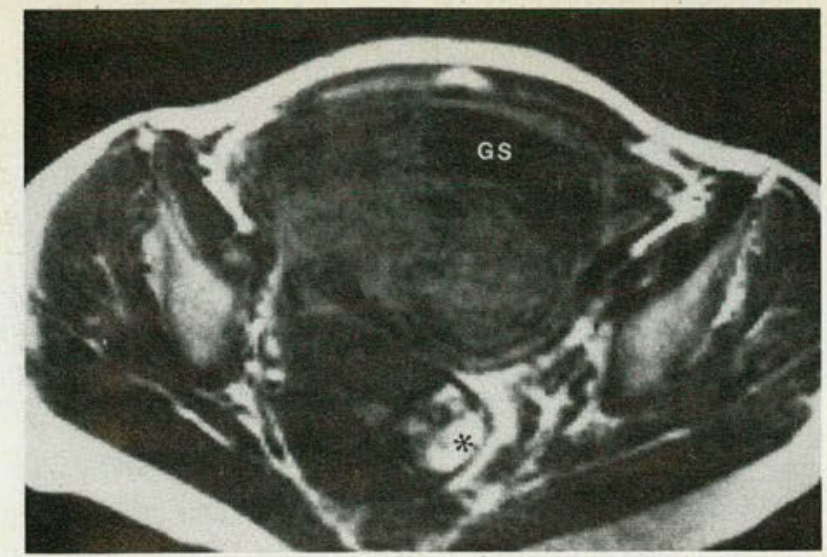

Figs 2,3. T1-weighted (700/33 msec) MRI: Transverse (Fig 2) and midline sagittal (Fig 3) images confirm the US findings, and, in addition, demonstrate a mural nodule (*) containing regions of high and low signal, which suggest the presence of fat and calcium $(B L=$ bladder and $G S=$ gestational sac; case 1$)$.

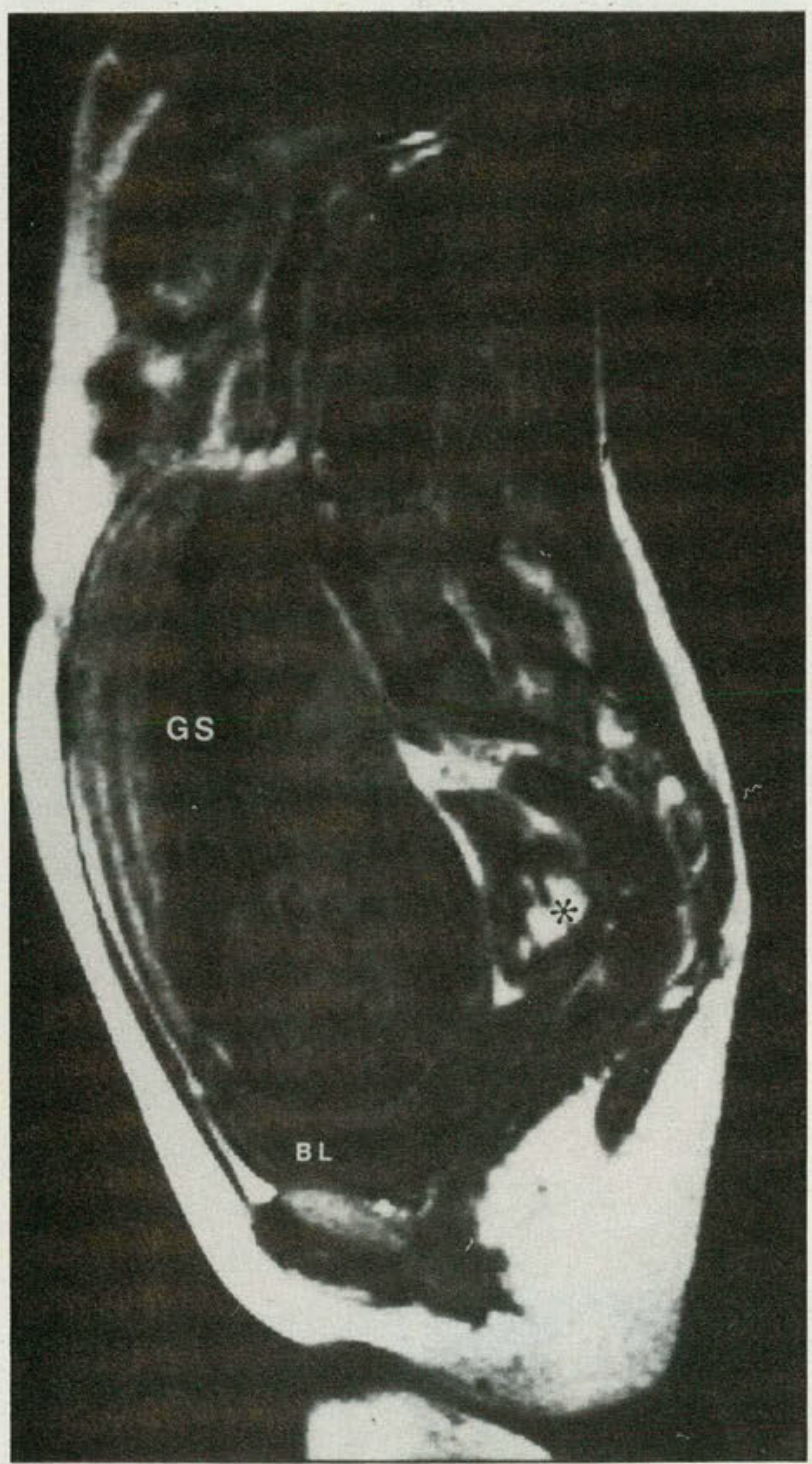

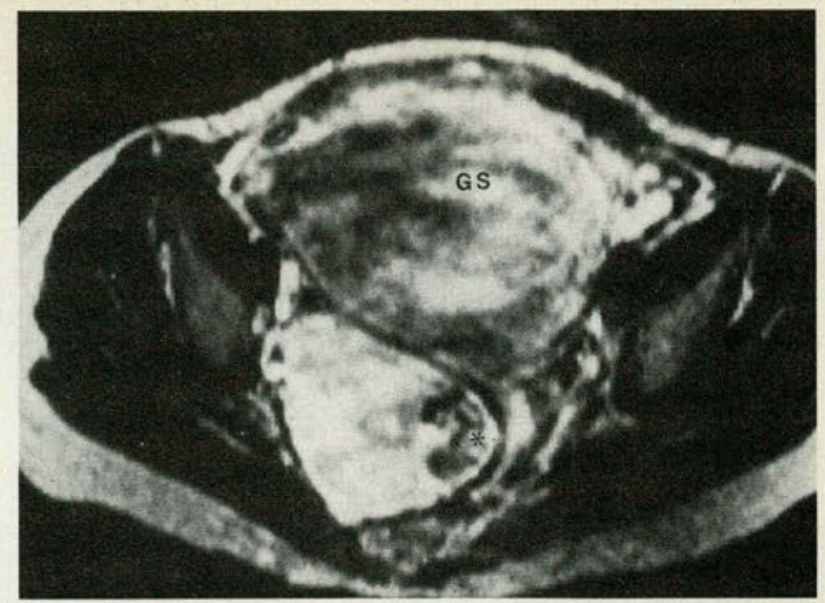

Fig 4. T2-weighted (2,000/100 msec) MRI: Transverse image again demonstrates a mural nodule (*). Signal characteristics of the cystic component are similar to amniotic fluid and urine (GS = gestational sac; case 1$)$.

tion thickness. The imaging matrix was $128 \times 256$ for two women and $192 \times 256$ for one woman.

Operative confirmation was obtained for two patients two and eight days after MRI. One patient did not undergo surgery.

\section{Case 1}

A 17-year-old girl (gravida 1, para 0 ) was found to have a 9-cm right ovarian mass at the time of her first obstetric evaluation by her private physician. Her prenatal history was unremarkable, except for irregular menses.

On physicial examination, the fundal height was compatible with an 18-week gestation. A right-sided pelvic mass was palpated, but it could not be delineated from the uterus.

Four weeks later, US demonstrated a $6.8 \times 5.9-\mathrm{cm}$ cystic mass in the cul-de-sac to the right of the midline (Fig 1). The full extent of the mass could not be evaluated because of its location. No fetal abnormalities were demonstrated. Estimated gestational age was 21 weeks. MRI confirmed the cul-de-sac mass and suggested the diagnosis of ovarian teratoma (Figs 2-4).

Because our experience with MRI was limited at this time, and because of the possibility of soft-tissue dystocia or tumor torsion during labor, exploratory laparotomy was performed the following day.

A $6 \times 8$-cm benign cystic teratoma of the right ovary was resected. The mural nodule demonstrated by MRI contained foci of fat, hair, and teeth. The patient's postoperative recovery was uneventful, and she was delivered of a healthy baby girl three months after her MRI examination.

\section{Case 2}

A 27-year-old woman (gravida 2, para 0, abortus 1) complained of a dull, right-sided pelvic pain that had been present since she had become pregnant. US early in her pregnancy had shown a large cul-de-sac mass. A second 
US examination at a different hospital demonstrated bilateral, 5- to 6-cm, adnexal masses and a single intrauterine pregnancy.

The patient was admitted to NHB for further evaluation. Her prenatal history was unremarkable. Physical examination demonstrated diffuse tenderness to deep palpation in the right lower quadrant. The uterus was 16 weeks' size. Bilateral adnexal masses were palpated; these were difficult to distinguish from the uterus. The uterus was felt to fill the cul-de-sac.

The next day, US revealed multiple uterine fibroids, without evidence of separate adnexal or cul-de-sac masses. A single fetus with an estimated gestational age of 15 weeks was present. No fetal abnormalities were present. Because of the strong clinical suspicion for bilateral adnexal masses, MRI study was performed (Figs 5-7). This confirmed the findings of US, and an exploratory laparotomy was averted. The patient was delivered of a healthy baby boy five months after her MRI examination.

\section{Case 3}

A 39-year-old woman (gravida 3, para 1, abortus 1) was found by US examination to have a 5 -cm, cystic, rightsided pelvic mass at the time of her initial obstetric evaluation. The patient's past medical history was significant for a 10-year history of Crohn's disease, for which she was receiving Azulfidine.

Repeat US 1 month later again demonstrated a teardrop-shaped, cystic, right-sided pelvic mass immediately beneath the terminal ileum. It had not changed in size in the interim. No peristaltic activity was observed in the region of the terminal ileum, but the bowel wall was not thickened. There was a single fetus of 17 weeks' age. No fetal abnormalities were seen.

Because of the patient's Crohn's disease and the relationship of the adnexal mass to the terminal ileum, MRI was performed (Fig 8-11).

Although neither the US or MRI examinations were suggestive of ovarian malignancy, this possibility could not be excluded in this older patient, and exploratory laparotomy and right ovarian cystectomy were performed eight days after the patient's MRI examination. The terminal ileum was normal to visual inspection at surgery. Pathologic examination of the mass revealed a benign serous ovarian cyst.

The patient had an uneventful postoperative recovery, but she delivered a grossly normal stillborn male infant in the 33rd week of pregnancy. Pathologic examination of the fetus was unremarkable.

\section{Discussion}

Ovarian tumors in pregnancy are not rare, with reported incidence ranging between 1 in 591 to 640 pregnancies. ${ }^{1,4}$ Although the most common masses occurring during pregnancy (corpus-luteum cyst of pregnancy, benign ovarian teratoma, and uterine leiomyoma) are nonmaligant, approximately $2 \%$ are cancerous. ${ }^{1,3,5,6}$ Other possible complications of pregnancy include torsion, rupture, soft-tissue

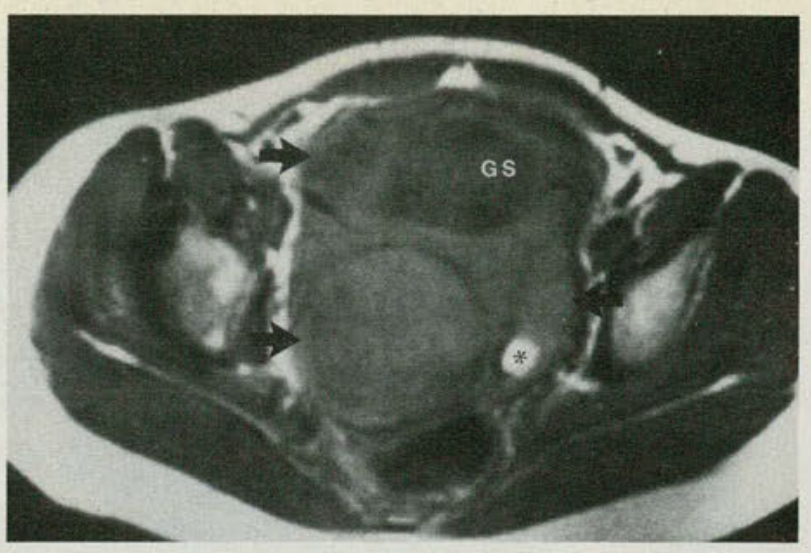

Fig 5. T1-weighted (700/33 msec) MRI: Transverse image demonstrates the gestational sac (GS) and multiple uterine leiomyomas (arrows). Area of increased signal on the left (*) represents a region of degeneration (case 2 ).

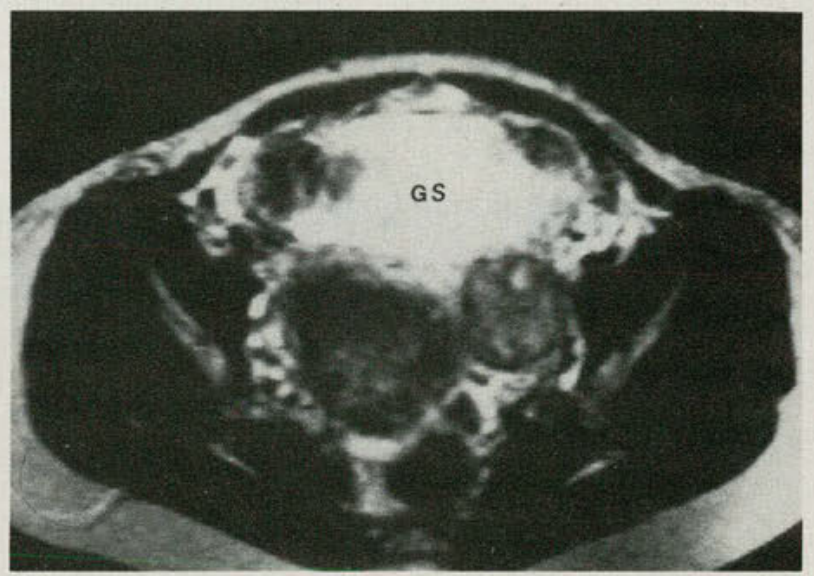

Figs 6,7. T2-weighted (2,000/100 msec) MRI transverse (Fig 6) and sagittal (Fig 7) images: T2 weighting increases the contrast between the leiomyomas and myometrium $(B L=$ bladder and GS = gestational sac; case 2).

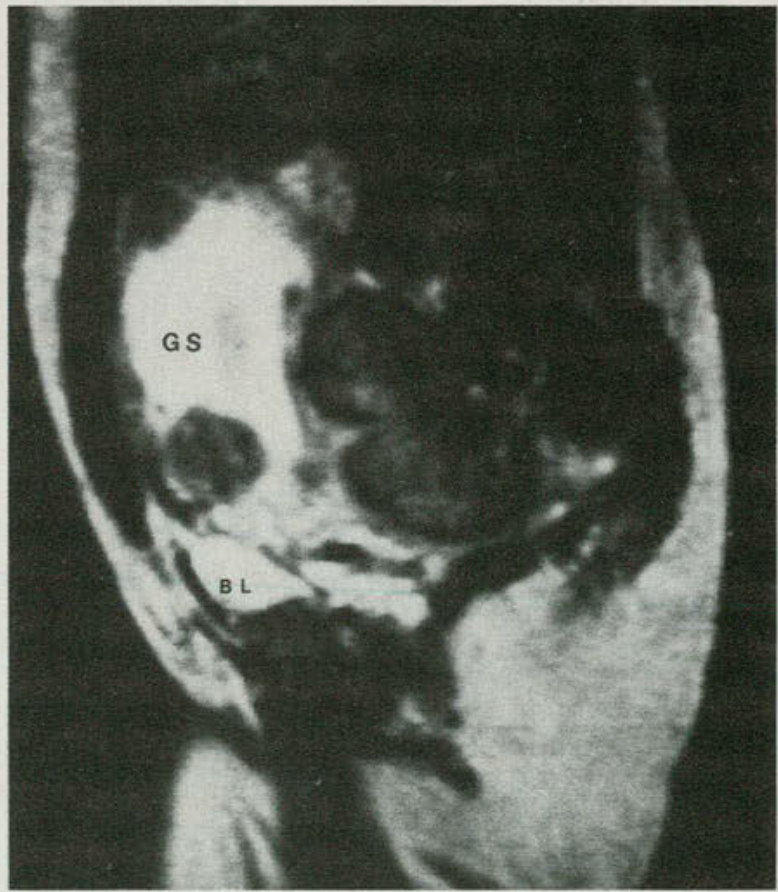




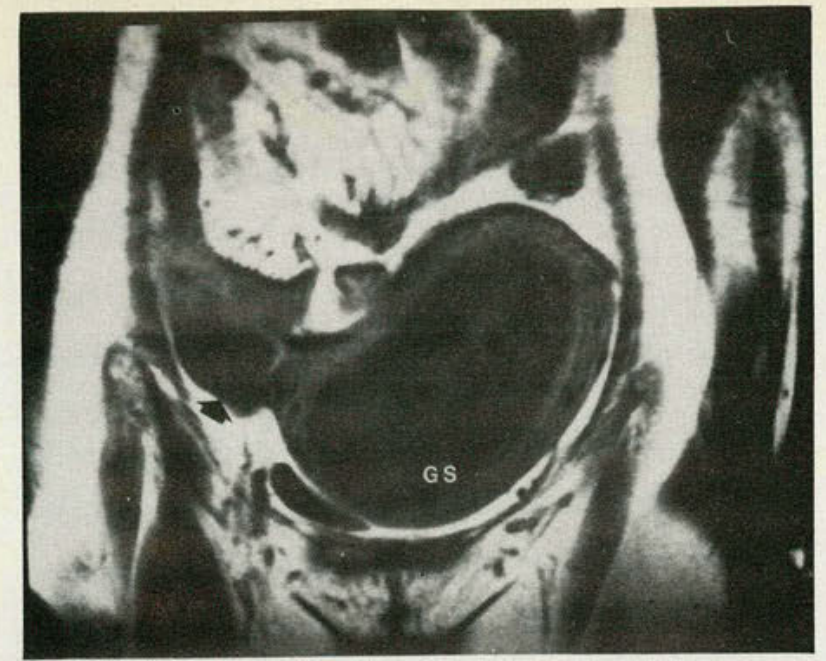

Figs 8-10. T1-weighted (700/33 msec) MRI: A cystic right adnexal mass (arrows) immediately inferior to but separate from the terminal ileum is seen in the coronal (Fig 8), transverse (Fig 9), and sagittal (Fig 10) planes (GS = gestational sac; case 3 ).
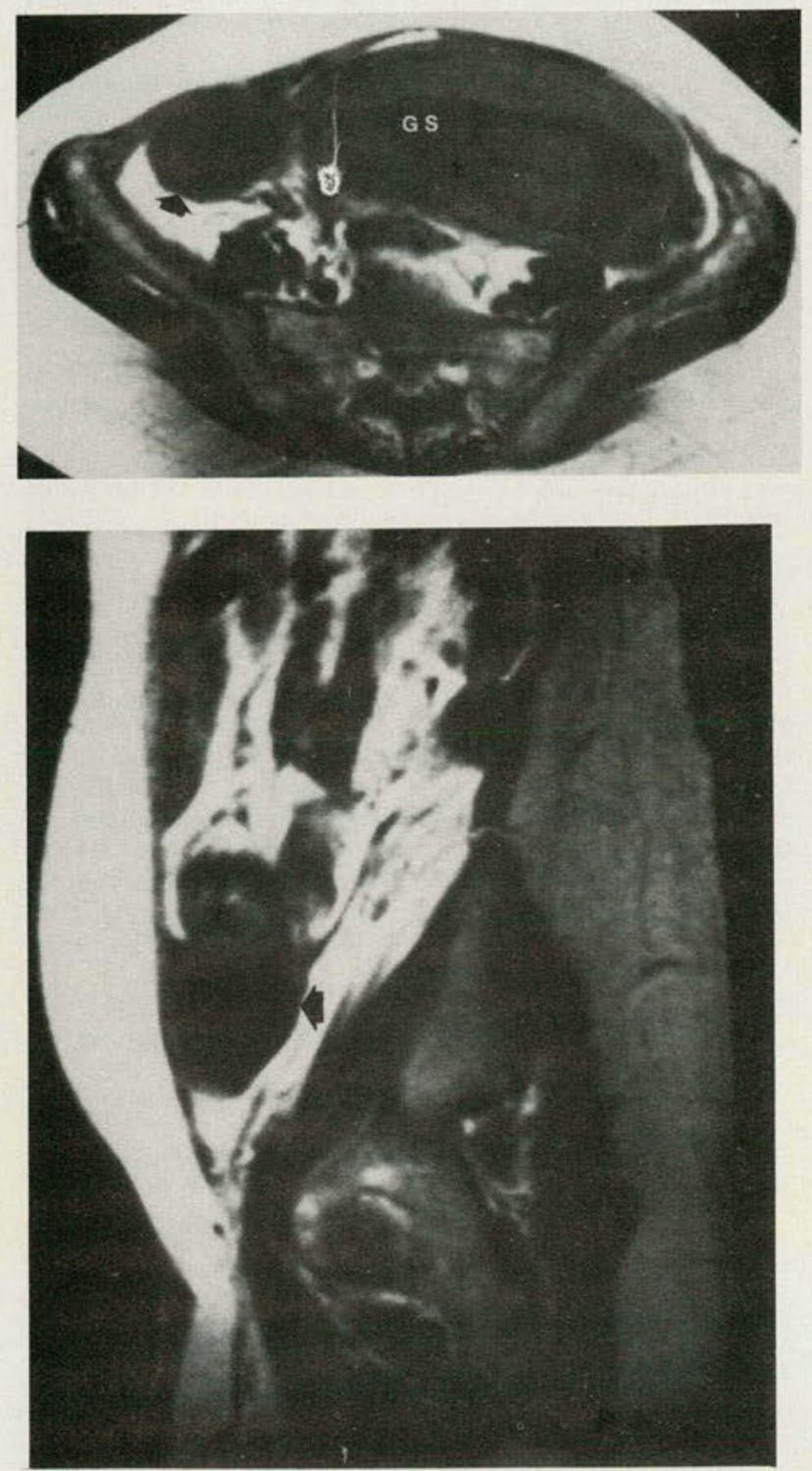

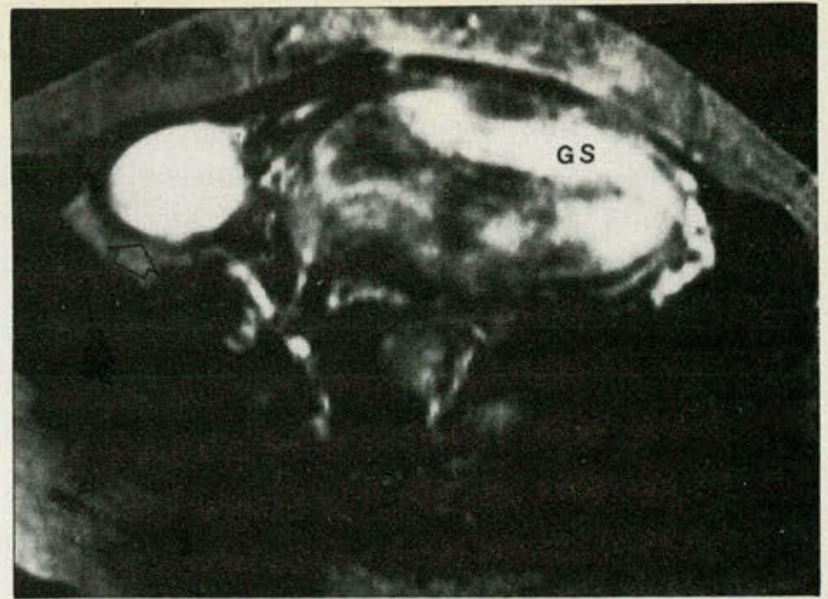

Fig 11. T2-weighted (2,000/100 msec) MRI: Transverse image at the same level as Fig 9 confirms the cystic character of the mass (open arrow) (GS=gestational sac; case 3 ).

dystocia during labor, infection, and bleeding. ${ }^{1,5,6}$ The obstetrician requires knowledge of the size and nature of the mass and the gestational age and wellbeing of the fetus to determine a therapeutic approach.

US is a valuable, proved technique for the detection and characterization of pelvic masses. ${ }^{7}$ It is simple, safe, inexpensive, and, currently, the only method for reliably imaging the fetus early in gestation. We agree with Weinreb and colleagues ${ }^{8}$ that sonography should be the initial imaging evaluation of all patients suspected of having a pelvic mass during pregnancy. However, the information obtained from US may be limited by the operator's skill or patient factors, and there is a need for a safe, complementary imaging technique for the obstetric patient..$^{9,10}$

MRI compares favorably with other imaging techniques in the evaluation of pelvic anatomy and pathologic conditions, is not operator-dependent, and surpasses other techniques in its ability to image in multiple planes. . $^{11-14}$ The signal characteristics of fat and blood also provide MRI with a degree of tissue characterization that is not possible with US.

In case 1 , the extent of the tumor was better evaluated by MRI. Also, the finding of increased signal on the T1-weighted sequence and decreased signal on the T1- and T2-weighted sequence in a mural nodule indicated the presence of fat and calcium, respectively, which allowed us to suggest the diagnosis of ovarian teratoma.

MRI confirmed the US impression of multiple uterine leiomyomas and absence of an adnexal mass in case 2 .

MRI also was helpful in localizing the origin of 
the mass in case 3 , although it provided no additional information to the US findings concerning the internal architecture of the mass.

MRI is very accurate in the diagnosis of uterine leiomyomas, ${ }^{12}$ but its specificity in the diagnosis of adnexal masses, except for teratomas, appears to be limited. ${ }^{11,15}$ Lack of specificity in the diagnosis of gynecologic masses is also a deficiency of US. ${ }^{7}$

MRI of the fetus currently is limited because of motion artifact, which is due to the time required to obtain a satisfactory image. However, useful information may be obtained in selected cases. ${ }^{9,10,16}$ Because the long-term effects upon the fetus are unknown, ${ }^{17-20}$ it has been suggested ${ }^{18}$ that MRI be avoided during the first trimester of pregnancy.

Because the most common adnexal mass in the first trimester is corpus luteum cyst of pregnancy, ${ }^{5}$ and because elective surgery on pelvic masses in pregnancy is delayed until the early part of the second trimester to increase fetal survivability, ${ }^{21}$ delaying MRI evaluation until after the 15 th week of pregnancy is a prudent and practical policy. Suspicion of ectopic gestation, molar pregnancy, or malignancy may be a valid reason for MRI earlier in pregnancy. 4,9

\section{Conclusion}

Our limited experience with three cases suggests that MRI is a complement to US in the evaluation of the patient whose pregnancy is complicated by a pelvic mass.

The opinions and assertions in this paper are the private views of the authors and are not to be construed as being official or as reflecting the views of the Departments of the Navy or Army or the Department of Defense.

1. Struyk APHB, Treffers PE: Ovarian tumors in pregnancy. Acta Obstet Gynecol Scand 1984;63:421-424.

2. Fleischer AC, Boehm FH, James AC Jr: Sonographic evaluation of pelvic masses and maternal disorders occurring during pregnancy, in Sanders RC, James AE Jr (eds): The Principles and Practice of Ultrasonography in Obstetrics and Gynecology. Norwalk, Conn, AppletonCentury-Crofts, 1985, pp 435-447.

3. Bezjian AA: Pelvic masses in pregnancy. Clin Obstet Gynecol 1984;27:402-415.

4. Kulkarni MV, Shaff MI, Carter MM, et al: Magnetic resonance imag- ing of the pelvic. Radiographics 1985;5:611-625.

5. McGowan L: Surgical diseases of the ovary in pregnancy. Clinical Obstet Gynecol 1983;26:843-852.

6. Ballard CA: Ovarian tumors associated with pregnancy termination patients. Am J Obstet Gynecol 1984;149:384-387.

7. Walsh JW, Taylor KJW, Wasson JFM, et al: Gray-scale ultrasound in 204 proven gynecologic masses: Accuracy and specific diagnostic criteria. Radiology 1979;130:391-397.

8. Weinreb JC, Brown CE, Lowe TW, et al: Pelvic masses in pregnant patients: MR and US imaging. Radiology 1986;159:717-724.

9. Weinreb JC, Lowe TW, Santos-Ramos R, et al: Magnetic resonance imaging in obstetrical diagnosis. Radiology 1985;154:157-161.

10. McCarthy SM, Filly RA, Stark DD, et al: Magnetic resonance imaging of fetal anomalies in utero: Early experience. AJR 1985;145:677682.

11. Dooms CG, Hricak H, Tscholakoff D: Adnexal structures: MR imaging. Radiology 1986;158:639-646.

12. Hricak H, Tscholakoff D, Heinrichs L, et al: Uterine leiomyomas: Correlation of MR, histopathologic finding, and symptoms. Radiology 1986;158:385-391.

13. Hricak $\mathrm{H}$, Alpers C, Crooks LE, et al: Magnetic resonance imaging of the female pelvis: Initial experience. AJR 1983;141:1119-1128.

14. Butler H, Bryan PJ, LiPuma JP, et al: Magnetic resonance imaging of the female pelvis. AJR 1984;143:1259-1266.

15. Bryan PJ, Butler HE, LiPuma JP: Magnetic resonance imaging of the pelvis. Radiol Clin North Am 1984;22:897-915.

16. Stark DD, McCarthy SM, Filly RA, et al: Interauterine growth retardation: Evaluation by magnetic resonance. Radiology 1985;155:425427.

17. Wolff S, Crooks LE, Brown P, et al: Tests for DNA and chromosomal damage induced by nuclear magnetic resonance imaging. Radiology 1980;136:707-710.

18. The National Radiological Protection Board Ad Hoc Advisory Group on Nuclear Magnetic Resonance Clinical Imaging: Revised guidance on acceptable limits of exposure during nuclear magnetic resonance clinical imaging. Br J Radiol 1983;56:974-977.

19. Budinger TF: Nuclear magnetic resonance (NMR) in vivo studies: Known thresholds for health effects. J Comput Assist Tomogr 1981;5:800811.

20. Geard CR, Osmak RS, Hall EJ, et al: Magnetic resonance and ionizing radiation: A comparative evaluation in vitro of oncogenic and genotoxic potential. Radiology 1984;152:199-202.

21. Saunders P, Milton PJD: Laparotomy during pregnancy: An assessment of diagnostic accuracy and fetal wastage. Br Med $J$ 1973;3:165167.

From the Department of Radiology (Dr Lubbers, Dr Goff, and Dr Johnson), Naval Medical Command-National Capital Region, Naval Hospital Bethesda, Bethesda, Md. From the Department of Radiology (Dr Lubbers, Dr Goff, Dr Johnson, and Dr Sikes), Uniformed Services University of the Health Sciences, Bethesda, Md. From the Department of Obstetrics and Gynecology (Dr. Izuno), Naval Medical Command-National Capital Region, Naval Hospital Bethesda, Bethesda, Md. From the Department of Radiology (Dr Sikes), Walter Reed Army Medical Center, Washington, DC.

Reprint requests to Dr Lubbers, Radiology Associates of Thomasville, P.C., P.O. Drawer 2450, Thomasville, GA 31799. 